\title{
Surface excitations in thin helium films on silica aerogel
}

\author{
H.J. Lauter ${ }^{1, *}$, I.V. Bogoyavlenskii ${ }^{2}$, A.V. Puchkov ${ }^{2}$, H. Godfrin ${ }^{3}$, A. Skomorokhov ${ }^{2}$, J. Klier ${ }^{4}$, P. Leiderer ${ }^{4}$ \\ ${ }^{1}$ Institut Laue Langevin, B.P.156, 38042, Grenoble Cedex 9, France \\ ${ }^{2}$ Institute for Physics and Power Engineering, 249020, Bondarenko sq.1, Obninsk, Russia \\ ${ }^{3}$ CNRS-CRTBT, 38042 Grenoble Cedex 9, France \\ ${ }^{4}$ Fachbereich Physik, Universität Konstanz, 78434 Konstanz, Germany
}

\begin{abstract}
First measurements are reported on pure surface excitations in thin superfluid ${ }^{4} \mathrm{He}$ films on silica aerogel. The ripplon dispersion curve is found to be the same for helium on graphite and silica aerogel substrates. However, the layered roton line width in helium films on aerogel shows a pronounced broadening with respect to that found in helium films on graphite.
\end{abstract}

PACS: 67.40.-w; 61.12.Ex

Surface excitations were first measured on graphite $[1,2]$ and modelled using a many-body theory [3]. The excitations present in very thin films are composed of ripplons and layered rotons. The ripplon is an excitation of the liquid-gas interface of the film, while the layered roton is an excitation bound to the layered structure of the liquid film itself. In the case of a very thin film only one layered structure and one layered roton exist adjacent to the solid-liquid interface. The liquid film is formed on top of a solid helium layer: on graphite, for instance, firstly two solid layers of helium are formed upon condensing helium in the sample. These solid layers also exist on aerogel substrate, but they are strongly modified due to the heterogeneous surface of this substrate. On a given substrate one can find a coverage of helium at which only the two above-mentioned excitations, ripplons and layered rotons, exist, i.e. no bulk excitations, e.g. phonons, maxons or rotons are observed. The ripplon dispersion relation is determined by the liquid-gas-interface properties, and therefore it shows essentially the same behaviour on graphite and on silica aerogel. On the other hand, the properties of the layered roton are expected to be affected by the nature of the solid-liquid interface; they are most conveniently determined at this particular coverage. The layered roton gap energy is determined by the substrate-helium interaction [3]; the curvature reflects its two-dimensional character through the liquid structure factor and the effective mass [3,4]; and its line width is anomalously broad. The last feature may have importance in relation to the bulk helium behaviour in aerogel, seen

*Corresponding author. (Fax: +33-476/207120, E-mail: lauter@ill.fr) when the aerogel is filled with helium, because the temperature dependence of the superfluid component shows near the lambda transition a different exponent with respect to helium in graphite [5].

\section{Experimental data}

The two-dimensional intensity plot of Fig. 1 shows the experimental dynamical structure factor of a thin helium film on aerogel measured at a temperature of $T=0.45 \mathrm{~K}$ at the time-of-flight (TOF) spectrometer IN6 [6] with an incident wavelength of $4.6 \AA$. At low momentum transfer $Q$ the ripplon is seen and at high $Q$ the layered roton. The much larger line width of the layered roton with respect to the one of the ripplon is apparent. The line width of the ripplon is determined by the instrumental resolution, which is in this region of energy transfer $\sim 0.2 \mathrm{meV}$. The helium coverage was chosen so that just no bulk roton signal is detectable. This means that approximately two layers of helium are liquid, if a comparison to helium on graphite is permitted [2] despite the 'rough' surface of aerogel. In order to obtain Fig. 1 a background was subtracted from the measurement. The background measurement consisted of the intensity scattered from the sample filled with $3400 \mathrm{cc}$ STP of condensed helium. The measurement itself was then performed with $4900 \mathrm{cc}$ STP of helium. A 'knee' in the adsorption isotherm, on a flat substrate a sign of a layer completion, taken at a temperature of $4 \mathrm{~K}$ shows up at a coverage of $1800 \mathrm{cc}$ STP of helium. So, in the balance there is a certain amount of non-superfluid liquid helium even if during cooling to $0.45 \mathrm{~K}$ more helium will solidify.

In Fig. 2 constant scattering angle scans are shown through $Q=1.9 \AA^{-1}$ and an energy transfer of $0.8 \mathrm{meV}$ performed at the DIN-2PI TOF spectrometer [7] with an incoming energy of $2.58 \mathrm{meV}$ at a temperature of the sample of $T=1.55 \mathrm{~K}$ (the resolution at this position is $0.15 \mathrm{meV}$ ). Here the He coverage is such that just a small signal of the bulk roton is visible. This signal of the bulk is fading for lower $Q$ and disappears below $Q=1.7 \AA^{-1}$. Such an effect typical for thin films has already been observed for helium on graph- 


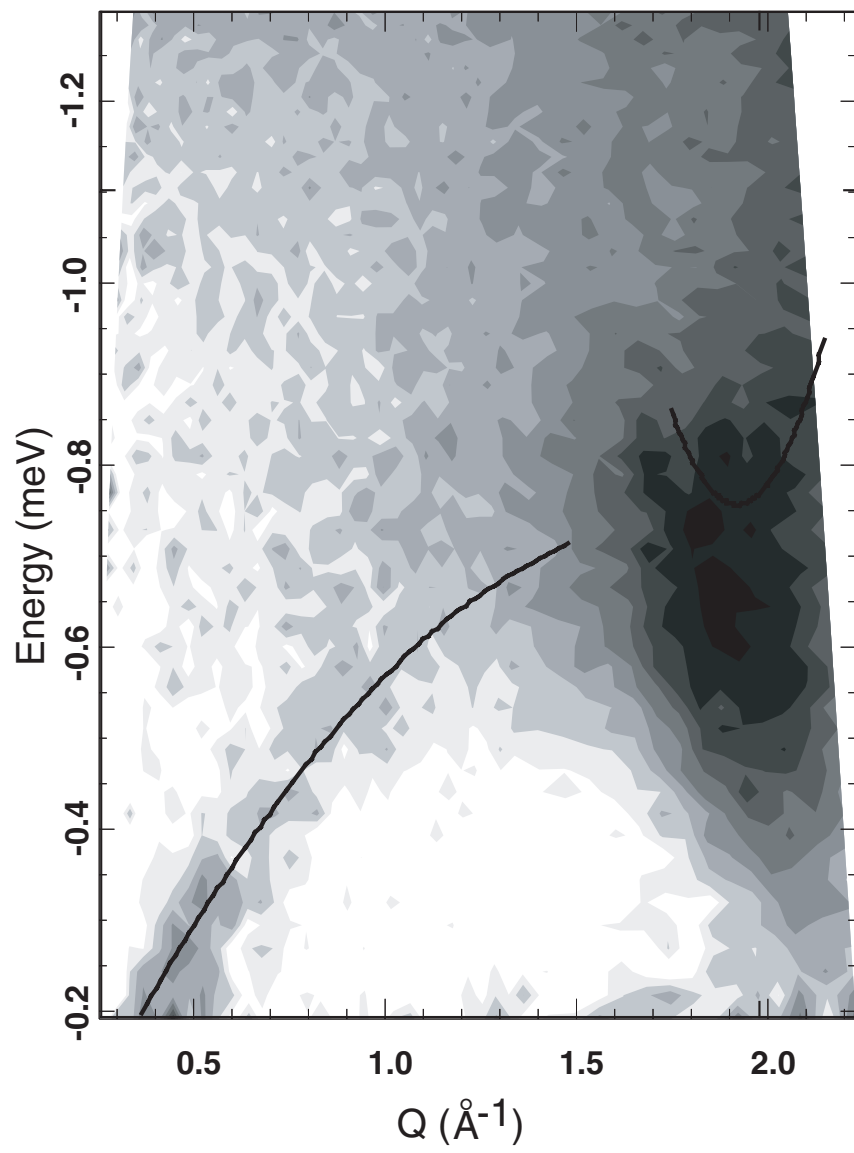

Fig. 1. Experimental dynamical structure factor $S(Q, \omega)$ (intensity, in grey contours, as a function of energy and momentum transfer) of a thin film of helium on aerogel substrate at a temperature of $T=0.45 \mathrm{~K}$ measured at IN6 [6]. From the origin the ripplon branch is rising and merges at around $Q=1.25 \AA^{-1}$ with the extension of the layered roton, the minimum of which is centred on $Q=1.95 \AA^{-1}$ and $E=0.63 \mathrm{meV}$. No bulk roton is visible. For comparison, the ripplon dispersion [2] is marked as a solid line as well as the bulk roton dispersion around its minimum

ite [2]. The signal of the layered roton, however, persists for lower $Q$ and runs over to the ripplon signal as discussed in the following Fig. 3. The second scan in Fig. 2 has been done

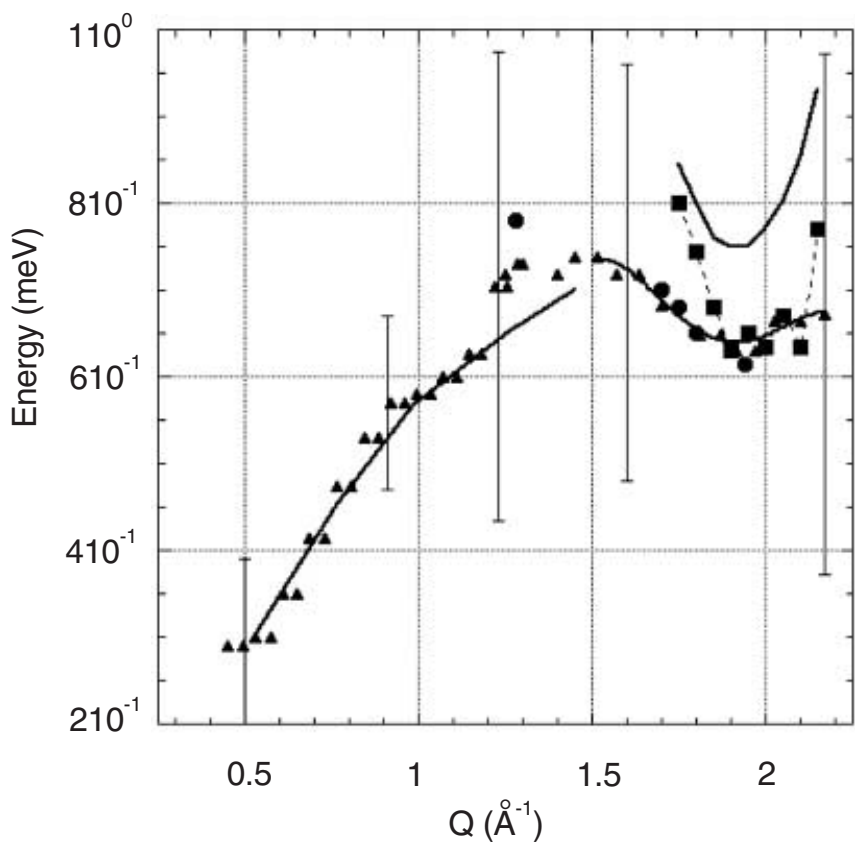

Fig. 3. Dispersion curves from the measurements shown in Figs. 1 and 2. Ripplon (black triangle) and layered roton have been measured on IN6 [6] and the layered roton $(\bullet)$ on DIN-2PI [7]. The data (black squares) are taken from [8]. For comparison, the dispersion of the ripplon [2] and the bulk roton have been added as solid lines. The FWHM of the line width (not the statistical error bar!) of the layered roton and the ripplon are shown for the IN6 data as error bars

with a $17 \%$ higher coverage, showing an increase of the intensity for the bulk roton and for the layered roton as seen for helium on graphite [2] or aerogel [8]. The line width of the bulk roton $(0.16 \mathrm{meV})$ is in agreement with the resolution of the instrument and the lifetime of the roton at $T=1.55 \mathrm{~K}$ $(\sim 0.04 \mathrm{meV})$. This coincides with the line width found for helium confined in Vycor [9] and shows that the line width of the roton is influenced by a restricted geometry. An other important result is that the line width of the layered roton of $\sim 1.1 \mathrm{meV}$ is doubled with respect to the line width at low temperature of $T=0.45 \mathrm{~K}$, which will be discussed in the next section.

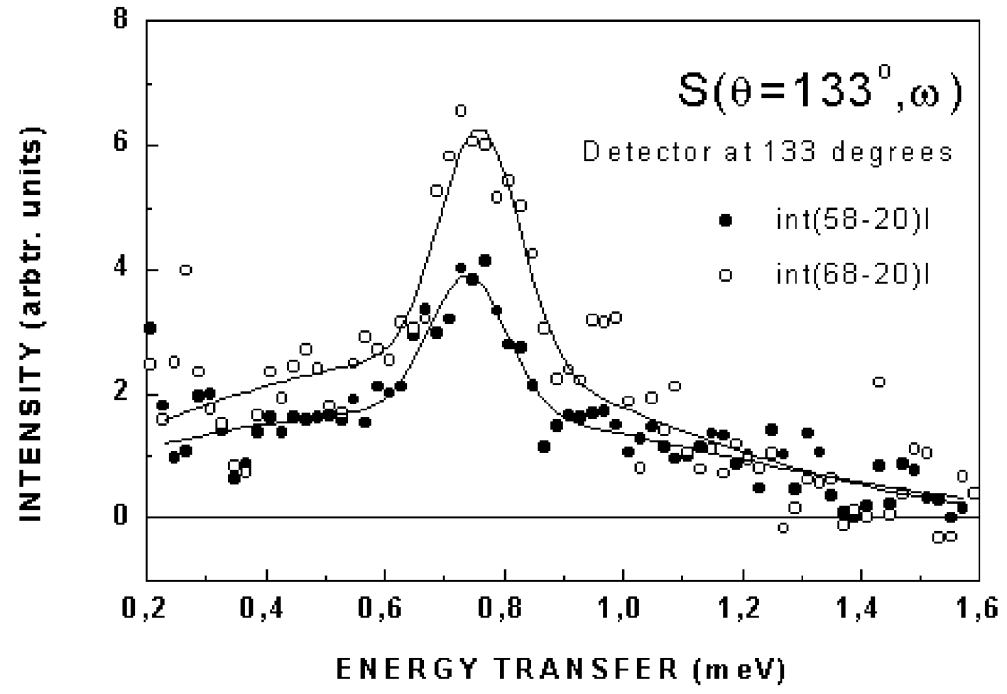

Fig. 2. Experimental dynamical structure factor; the scans performed for two coverages cross the bulk roton minimum at $Q=1.9 \AA^{-1}$ (bulk roton). The coverage on top of the aerogel substrate is • $581 \mathrm{STP}$ of helium and $\circ 681 \mathrm{STP}$ of helium A background, showing no signal from the helium on dispersion curves with 201 STP of helium, has been subtracted. The bulk roton and the layered roton are fitted by Gaussian profiles 


\section{Results and discussion}

The ripplon dispersion, shown in Fig. 3 (see also Fig. 1), follows the known behaviour from superfluid helium on graphite [2], and thus effects from a different substrate show no influence on the liquid-gas interface, which is determinative for the ripplon. The line width of the ripplon is governed by the instrumental resolution.

We will not discuss the few points measured on the bulk roton curve. The bulk dispersion is shown only around the roton minimum for comparison to the layered roton dispersion.

The layered roton exhibits a minimum (see Fig. 3) at the same value of $0.64 \mathrm{meV}$ as in [8]; however the dispersion is different. Here we find from both measurements a much more shallow curvature around the minimum. This agrees with the basic argument of a wider first maximum of the two-dimensional structure factor with respect to three dimensions [4] verified in the model calculation [3]. The model calculation also predicts a rather smooth crossover to the ripplon as seen in Figs. 3 and 1. The large line width of the layered roton compared to the one of the ripplon is astonishing. For the discussion of this effect we have to examine the data of the structure factor [10] of low-coverage films of helium on aerogel. When a first amount of helium is condensed onto the aerogel equivalent to less than a monolayer on graphite, the measured structure factor shows no Bragg peaks but a broad hump at low momentum transfer. This feature resembles a first maximum of the structure factor of a low-density liquid not reaching the density of bulk liquid helium. However, due to the interaction potential between the helium atoms and the aerogel surface the helium atoms adjacent to the aerogel must be tightly bound. Thus a solid structure is possible with no long-range order building cavities nucleating from the aerogel surface. Upon condensing further amounts of helium this structure with cavities is compressed without losing its character. This is deduced from the fact that upon further helium condensation the hump in the structure factor is moving to higher momentum-transfer values. Then the signals of the ripplon and the layered roton appear, which are the signs of superfluidity. This growth of solid helium with cavities is obviously triggered by the aerogel surface. The helium restricted in these cavities may give rise to the extreme broadening of the line width of the layered roton. This is in line with the above-mentioned argument that a two-dimensional structure factor leads to a broadening of the line width. Thus a further restriction by a further confining geometry leads to a further broadening. The same argument is found in the picture of the density currents. The density currents of the layered roton calculated in [3] are localised at the solid helium-liquid helium interface with a restricted movement perpendicular to the surface. An additional lateral restriction due to 'cavities' at the solid-helium surface will restrict further the density currents and will lead to a broadening of the line width.

The line width of the layered roton measured in Vycor [9] is about half the one presented here on aerogel. This agrees with the smoother surface of the Vycor pores with respect to the rough surface of the aerogel composed of silica strands. Comparing the results from helium on aerogel of [8] to our results obtained also from helium on aerogel leads to the conclusion that the surface constitution of the aerogels used in the two experiments must be different. The energy gap of the layered roton minimum is in agreement; however the smaller line width of the layered roton of about $0.16 \mathrm{meV}$ [8] points to a smoother surface of the aerogel used in [8]. A smoother surface should lead to a more pronounced two-dimensional character of the layered roton as seen in the shallower minimum of the presented data (see Fig. 3). But, inexplicably, the data of [8] show in contrast a curvature very close to the one of the bulk roton. The layered roton of our data exhibits an even more increased line width at a temperature of $T=1.55 \mathrm{~K}$ (see Fig. 2), which amounts to about $2 \mathrm{meV}$; however a theoretical background to explain this behaviour as it is done for the bulk roton in a confining geometry [9] is still missing.

In conclusion, it was elaborated that the solid helium coating with cavities underneath the liquid helium film is responsible for the extreme line-width broadening of the layered roton. This explanation is compatible with a non-affected line width of the ripplon of a thin helium film adsorbed on aerogel. The two-dimensional character of the layered roton is underlined by the shallow dispersion around the layered roton minimum.

\section{References}

1. W. Tomlinson, J.A. Tarvin, L. Passell: Phys. Rev. Lett. 44, 266 (1980)

2. H.J. Lauter, H. Godfrin, V.L.P. Frank, P. Leiderer: Phys. Rev. Lett. 68, 2484 (1992); H.J. Lauter, H. Godfrin, V.L.P. Frank, P. Leiderer: In "Excitations in Two-Dimensional and Three-Dimensional Quantum Fluids", A.F.G. Wyatt, H.J. Lauter eds., Springer Series in Solid State Sciences Vol. 79, p. 149

3. B.E. Clements, H. Godfrin, E. Krotschek, H.J. Lauter, P. Leiderer, V. Passiouk, C.J. Tymczak: Phys. Rev. B 53, 12242 (1996); B.E. Clements, E. Krotschek, C.J. Tymczak: Phys. Rev. B 53, 12253 (1996)

4. W. Götze, M. Lücke, J. Low: Temp. Phys. 25, 671 (1976)

5. M.H.W. Chan, K.I. Blum, S.Q. Murphy, G.K.S. Wong, J.D. Reppy: Phys. Rev. Lett. 61, 1950 (1988)

6. http: //www.ill.fr/YellowBook/IN6/

7. http: //nfdfn.jinr.ru/

8. B. Fak, O. Plantevin, H.R. Glyde, N. Mulders: Phys. Rev. Lett. 85, 3886 (2000)

9. R.M. Dimeo, P.E. Sokol, C.R. Anderson, W.G. Stirling, K.H. Andersen, M.A. Adams: Phys. Rev. Lett. 81, 5860 (1998)

10. H. Godfrin, J. Klier, H.J. Lauter, P. Leiderer: ILL-Exp. Report 6-01171 (1998) 